\title{
Craqueamento Catalítico de Polietileno em Condições de Refinaria - Produção de Frações Combustíveis
}

\author{
Alessandra M. Ribeiro, Hélio F. Machado Júnior, Dilma A. Costa \\ Departamento de Engenharia Química, UFRRJ
}

Resumo: Este trabalho foi realizado em uma unidade de teste de microatividade para estudar o processo de craqueamento catalítico das cargas combinadas de polietileno de baixa densidade e polietileno de alta densidade com vaselina, frente a catalisadores comerciais de FCC (alta e baixa atividades), para avaliar a produção das frações combustíveis (gasolina, diesel e resíduo). As cargas combinadas de PEBD e PEAD/vaselina foram processadas em condições de refinaria. Para as cargas de PEBD/vaselina, a 2, 6 e 10\% p/p, a produção da fração gasolina foi favorecida pelo catalisador de alta atividade, enquanto que a carga de $\mathrm{PEAD} /$ vaselina a $2 \% \mathrm{p} / \mathrm{p}$, para produção da mesma fração, o catalisador de baixa atividade apresentou melhor eficiência. Todas as cargas combinadas, nas diversas concentrações, mostraram que o material inerte (caulim) apresenta maior atuação, na produção da fração resíduo, indicando a ocorrência preferencial de craqueamento térmico.

Palavras-chave: Polietileno, degradação, zeólita.

\section{Catalytic Cracking of Polyethylene under Refinery Conditions - Production of Combustible Fractions}

\begin{abstract}
This work was carried out in an unit of microactivity test, to study the process of combined feeds of low density and high density polyethylenes with vaseline and commercial FCC catalysts (of low and of high activities), to evaluate the production of fuel fractions (gasoline, diesel and residue). The combined feeds of PEBD and PEAD/vaseline, at different concentrations, were processed under refinery conditions. For feeds of PEBD/vaseline at 2, 6 and 10\% w/w, production of the gasoline fraction was favored with the high-activity catalyst, while for the PEAD/vaseline feed at $2 \%$, in the production of the same fraction, the low-activity catalyst presented better performance. For all the combined feeds, in all concentrations, the inert material showed better performance for the production of residue fraction, indicating the preferential occurrence of thermal cracking.
\end{abstract}

Keywords: PEBD, PEAD, fluid catalytic cracking, FCC catalyst, gasoline fraction.

\section{Introdução}

No Brasil, as poliolefinas representam cerca de $65 \%$ das materiais plásticas produzidas, onde os polietilenos correspondem a $43 \%$. Industrialmente podem ser produzidos diferentes tipos de polietileno, de acordo com as condições reacionais e com o sistema catalítico empregado na polimerização ${ }^{[1]}$.

O Polietileno (PE) é um polímero parcialmente cristalino, flexível, cujas propriedades sofrem influência da quantidade relativa das fases amorfa e cristalina ${ }^{[1,2]}$. O Polietileno de baixa densidade (PEBD) apresenta em sua estrutura ramificações, na maioria das vezes, tão longas quanto à cadeia principal do polímero. A presença dessas ramificações determina o grau de cristalinidade, as temperaturas de transição e afeta parâmetros cristalográficos, tais como o tamanho dos cristalitos $^{[1,3,4]}$. O Polietileno de alta densidade (PEAD) é linear e altamente cristalino (acima de 90\%), com baixo teor de ramificações. A linearidade das cadeias do PEAD torna a orientação, o alinhamento e o empacotamento das cadeias mais eficiente, fazendo com que as forças intermoleculares (do tipo Van der Waals), possam agir mais intensamente, e, consequentemente, aumentem sua cristalinidade em relação ao $\operatorname{PEBD}^{[1]}$.

O craqueamento catalítico de polímeros ocorre de maneira semelhante ao processo de craqueamento catalítico fluido (FCC) do gasóleo, onde hidrocarbonetos pesados são convertidos a produtos mais leves (C5 a C12), de alta qualidade. Esse tipo de craqueamento ocorre a temperaturas controladas e mais baixas que o craqueamento térmico ${ }^{[5-9]}$.

Os catalisadores já utilizados no craqueamento catalítico de polímeros foram: $\mathrm{Al}_{2} \mathrm{O}_{3} ; \mathrm{SiO}_{2}$; catalisadores de sílicaalumina amorfa e de sílica mesoporosa; SAPO-37; argilas; catalisadores zeolíticos - Y (NaY, HY e REY), HMOR, HZSM-5, HNZ, HUSY, HTHETA1, Beta, MFI, BEA; e catalisador de $\mathrm{FCC}^{[10-17]}$.

Os catalisadores de FCC possuem em sua composição diferentes teores de zeólita Y, como matéria prima principal. A zeólita Y é um aluminossilicato sintético, da família das faujasitas. Esse tipo de zeólita possui uma estrutura tridimensional caracterizada pela união entre octaedros truncados, através de prismas hexagonais ${ }^{[18,19]}$.

Desta forma, este trabalho teve como objetivo geral estudar a influência da estrutura molecular do PEBD e do PEAD,

Autor para correspondência: Dilma A. Costa, Departamento de Engenharia Química, IT, UFRRJ, BR-465, Km 07, CEP: 23890-000, Seropédica, RJ, Brasil. 
na composição das frações combustíveis (gasolina, diesel e resíduo), produzidas pelo craqueamento catalítico fluido (FCC) de cargas combinadas desses polímeros com vaselina (mistura de parafinas), em condições de refinaria, utilizando catalisadores comerciais de FCC (de baixa e alta atividade).

\section{Materiais e Métodos}

\section{Materiais}

Os materiais usados neste trabalho foram: PEBD (ABCM Plásticos LTDA); PEAD (Plasmar Ind e Com.); vaselina (Resim Indústria e Comércio LTDA/PETROBRÁS/BR); caulim - alumino-silicato (Referência da CADAM - Caulim da Amazônia S.A.) e catalisadores comerciais de FCC Fluid Catalytic Cracking (fornecidos pela Fábrica Carioca de Catalisadores - FCC S.A/RJ).

A vaselina (mistura de parafinas) foi utilizada como solvente e apresenta composição química de (95-99\%) de parafinas, $0,4 \%$ de aromáticos e $0,07 \%$ de compostos de enxofre. O caulim, material inerte, foi utilizado como padrão, branco, no craqueamento catalítico fluido.

Os catalisadores foram classificados de acordo com o percentual de zeólita Y (componente ativo) contido na composição comercial, como: Baixa Atividade (BA) e Alta Atividade (AA).

\section{Método utilizado no processo de craqueamento catalítico fluido}

Para este trabalho foi montada e utilizada uma unidade padrão de avaliação de microatividade (M.A.T.), tendo como base o Standart Test Procedure - ASTM D 3907 $7^{[20]}$. A montagem da unidade (M.A.T.) fundamentou-se em unidades-teste existentes na Fábrica Carioca de Catalisadores S.A., que podem ser utilizadas para pré-estabelecer variáveis, como: velocidade espacial, tempo de corrida, temperatura e carga. Neste trabalho foram obtidas cargas de diferentes concentrações, de acordo com cada polímero utilizado no teste catalítico e buscou-se reproduzir, de maneira mais real possível, as condições utilizadas em refinaria, conforme mostrado na Figura 1.

As amostras de catalisador comercial de FCC (BA e AA) foram ativadas "in situ", por $20 \mathrm{~min}$, na temperatura de reação $\left(520^{\circ} \mathrm{C}\right)$, sob fluxo de nitrogênio e a pressão atmosférica, de forma a eliminar qualquer resíduo de umidade existente.

As cargas combinadas dos polímeros PEBD e PEAD, com vaselina, foram obtidas nas concentrações de 2 e $4 \%$ p/p. Também foram obtidas cargas combinadas de PEBD/vaselina, nas concentrações 6 e 10\% p/p. Essas cargas foram processadas na unidade de teste (M.A.T.), frente ao caulim e aos catalisadores comerciais de FCC.

\section{Caracterização de análise dos polímeros e produtos de craqueamento}

Espectroscopia de infravermelho com transformada de Fourier (FTIR)

As estruturas moleculares das amostras de PEBD e PEAD foram caracterizadas pela técnica de espectroscopia na região do infravermelho com transformada de Fourier -

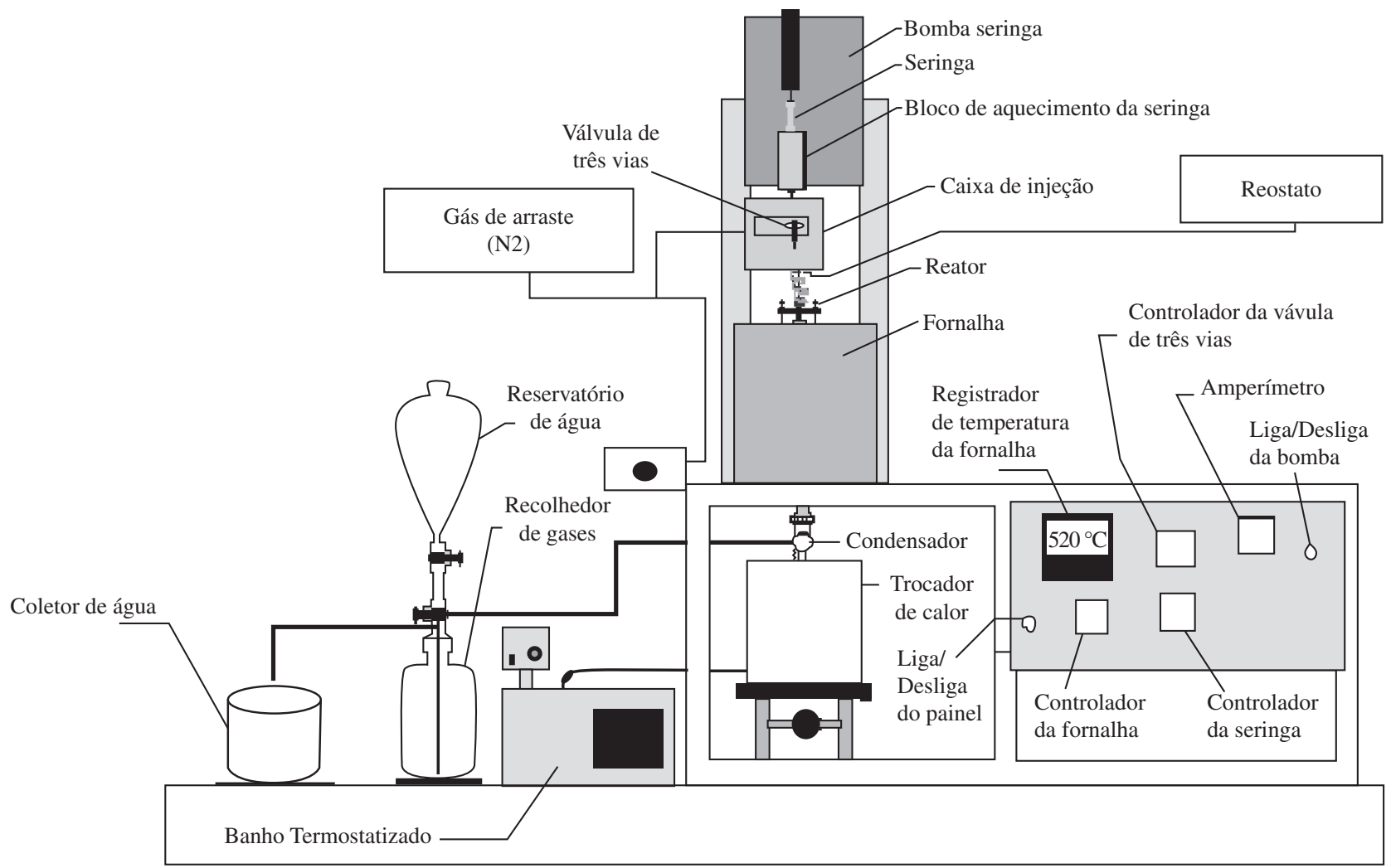

Figura 1. Unidade de Teste de Microatividade (M.A.T.). 
FTIR (Perkin Elmer, modelo FTIR-2000), a partir de filmes prensados de PEBD e PEAD, na faixa de número de ondas de 400 a $4000 \mathrm{~cm}^{-1}$, utilizando-se a técnica de reflexão interna múltipla (MIR), e calibração com padrão de poliestireno ${ }^{[21]}$. As absorções das bandas características dos grupos vinileno, vinil e vinilideno existentes nos polietilenos (PEBD e PEAD) foram utilizadas para construção de uma curva de calibração para estimar o peso molecular (Mn) do PEBD e do PEAD (Figura 2). Estas bandas estão presentes em números de ondas de $965 \mathrm{~cm}^{-1}, 908 \mathrm{~cm}^{-1}$ e $888 \mathrm{~cm}^{-1}$, respectivamente ${ }^{[21-23]}$.

As amostras de PEBD, PEAD e das frações líquidas produzidas também foram analisadas por FTIR, para confirmação da ocorrência do craqueamento catalítico.

\section{Cromatografia gasosa $(G C)$}

Os produtos do processo de craqueamento catalítico foram classificados como, líquidos, sólidos e gasosos. O produto líquido foi analisado por cromatografia gasosa em um cromatógrafo a gás da marca Varian e modelo 3300 Gas Chromatograph, com detector de ionização de chama (FID), acoplado a um microcomputador. O produto sólido foi analisado por determinação do teor de coque e a quantidade de produto gasoso foi calculada pela diferença do somatório de produtos líquido e sólido, não sendo analisada a sua composição.

$\mathrm{Na}$ cromatografia gasosa foi utilizada uma coluna capilar de sílica fundida CP-SIL-5 CB da Chrompack, de comprimento de $25 \mathrm{~m}$ e diâmetro interno de $0,25 \mathrm{~mm}$. A temperatura inicial da coluna foi de $60^{\circ} \mathrm{C}$, mantida por $2 \mathrm{~min}$. Foram estabelecidas condições de análise, de acordo com a literatura ${ }^{[21]}$.

O produto líquido da reação foi separado em faixas das frações, de acordo com os padrões utilizados pela indústria petroquímica. Para isso, foram injetados componentes puros e misturados, para a determinação dos tempos de retenção adotados como padrões internos, tais como, hexano $\left(\mathrm{C}_{6} \mathrm{H}_{14}\right)$, dodecano linear $\left(\mathrm{C}_{12} \mathrm{H}_{26}\right)$ e o eicosano $\left(\mathrm{C}_{20} \mathrm{H}_{42}\right)$. O padrão de FCC foi injetado com o objetivo de se obter a sobreposição dos tempos de retenção dos padrões internos, estabelecendo exatamente, o tempo de retenção de começo e término de cada faixa combustível produzida pelo craqueamento.

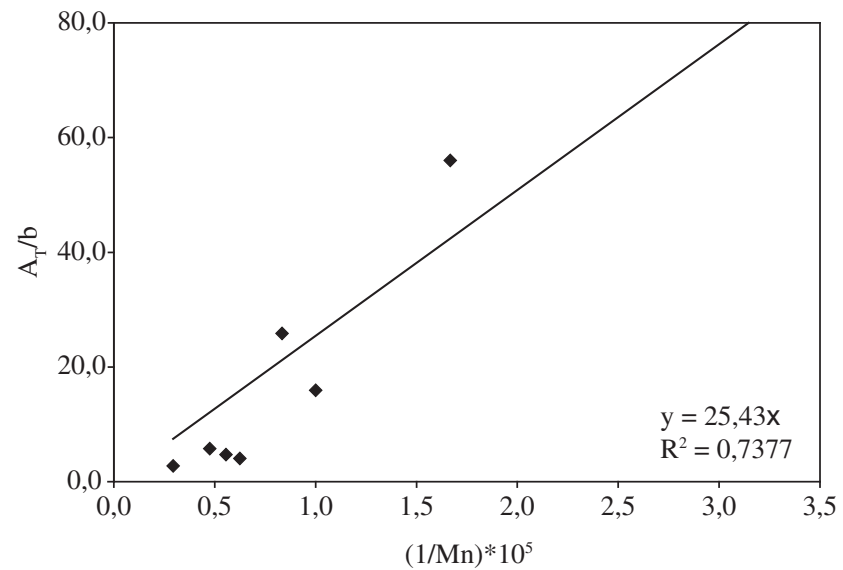

Figura 2. Curva de calibração do PE para estimar valores de Mn do PEBD e PEAD ${ }^{[21,22]}$.

\section{Determinação de coque}

Os produtos sólidos foram analisados pelo equipamento da marca LECO, modelo CS-125, para a determinação do teor de carbono (formação de coque), tanto na superfície do catalisador, quanto no interior de seus poros, após a reação. $\mathrm{O}$ percentual em peso de coque formado no catalisador de FCC, com o craqueamento, foi calculado de acordo com a Equação 1.

$$
\begin{aligned}
& \% \text { Coque } \mathrm{p} / \text { Carga }= \\
& \frac{\% \text { Coque LECO } * \mathrm{~m}(\mathrm{~g}) \text { catalisador }}{\mathrm{m}(\mathrm{g}) \text { carga }}
\end{aligned}
$$

onde: $\mathrm{m}(\mathrm{g})$ catalisador $=5,0 \mathrm{~g}$ e $\mathrm{m}(\mathrm{g})$ carga $=1,0 \mathrm{~g}$ (para um tempo de injeção de carga de 30 s). E, \%Coque para Carga = \%Produto Sólido.

\section{Diferença com líquido e sólido}

A quantidade de produtos gasosos foi obtida através da diferença do somatório de produto líquido e de produto sólido, sem especificação de suas composições. A determinação do percentual de produto gasoso gerado foi calculado a partir do cálculo do percentual de produto líquido e do percentual em peso de produto sólido gerado (coque), de acordo com a Equação 2.

$$
\begin{aligned}
& \text { \%Produto Líquido }= \\
& \frac{\mathrm{m}(\mathrm{g}) \text { Produto Líquido }+ \text { cotonete }}{\mathrm{m}(\mathrm{g}) \text { carga }} * 100
\end{aligned}
$$

onde: A massa do cotonete é a diferença considerada entre o peso, em gramas do cotonete limpo, e o peso do cotonete após a limpeza do reator.

\section{Resultados e Discussão}

\section{Espectroscopia de infravermelho com transformada de Fourier}

\section{Caracterização dos materiais}

A partir das áreas das bandas de vinileno $\left(965 \mathrm{~cm}^{-1}\right)$, vinil $\left(908 \mathrm{~cm}^{-1}\right)$ e vinilideno $\left(888 \mathrm{~cm}^{-1}\right)$, do PEBD e do PEAD e das espessuras de seus filmes foi possível normalizar as áreas totais e estimar os valores de Mn dos polímeros (PEBD e PEAD), de $6,0 \times 10^{4}$ para o PEBD e $6,5 \times 10^{5}$ para o PEAD.

As bandas mais características dos polímeros (PEBD e PEAD) estão apresentadas na Figura 3.

\section{Determinação do produto líquido por cromatografia gasosa}

As amostras de produtos líquidos foram analisadas por cromatografia gasosa com FID, para proporcionar a separação dos produtos em faixas, de acordo com padrões utilizados pela indústria petroquímica. Os hidrocarbonetos puros e misturados (hexano $-\mathrm{C}_{6} \mathrm{H}_{14}$, dodecano linear $-\mathrm{C}_{12} \mathrm{H}_{26}$ e 
eicosano $-\mathrm{C}_{20} \mathrm{H}_{42}$ ) foram utilizados como padrões internos, visando a demarcação dos limites dos tempos de retenção destes, dentro do cromatograma do padrão de FCC (contendo $50 \%$ de Gasolina (C5-C12), 30\% de Óleos Leves de Reciclo - LCO (C13-C20, contendo o Diesel) e 20\% de Resíduo (acima de $\mathrm{C} 20)$.

A partir da demarcação destes tempos de retenção foi possível calcular o percentual de cada fração (Gasolina, Diesel e Resíduo) correspondente a cada faixa. Os percentuais dos produtos líquidos do craqueamento da vaselina e das cargas combinadas de PEBD/vaselina (2, 4, 6 e 10\% p/p), na presença de caulim e dos catalisadores comerciais de FCC estão apresentados nas Figuras 4-6. Pode-se observar pela Figura 4, que o caulim (inerte) produz menor percentual da fração Gasolina do que os catalisadores zeolíticos e que a vaselina fornece os maiores percentuais de produtos de craqueamento, provavelmente pela maior susceptibilidade das cadeias parafínicas de sofrerem reação de craqueamento. Observa-se também que o catalisador comercial de alta atividade (AA) se mostra o mais eficiente na produção dessa fração, para todas as cargas combinadas craqueadas. À medida que a concentração de PEBD aumenta, na carga combinada, observa-se uma ligeira diminuição da produção da fração Gasolina (6\% p/p). No entanto, para cargas combinadas contendo baixas concentrações de PEBD (2\% p/p), o catalisador comercial de baixa atividade (BA) é suficiente para atingir um alto percentual de produção da fração Gasolina.

A Figura 5 mostra que de um modo geral o caulim (material inerte) se mostrou mais efetivo para a produção da fração Diesel, indicando a ocorrência preferencial de craqueamento térmico, e que as cargas combinadas de $\mathrm{PEBD} /$ vaselina produzem percentagens bem mais baixas da faixa de Diesel, em comparação com a faixa de Gasolina, para os 2 tipos de cata-

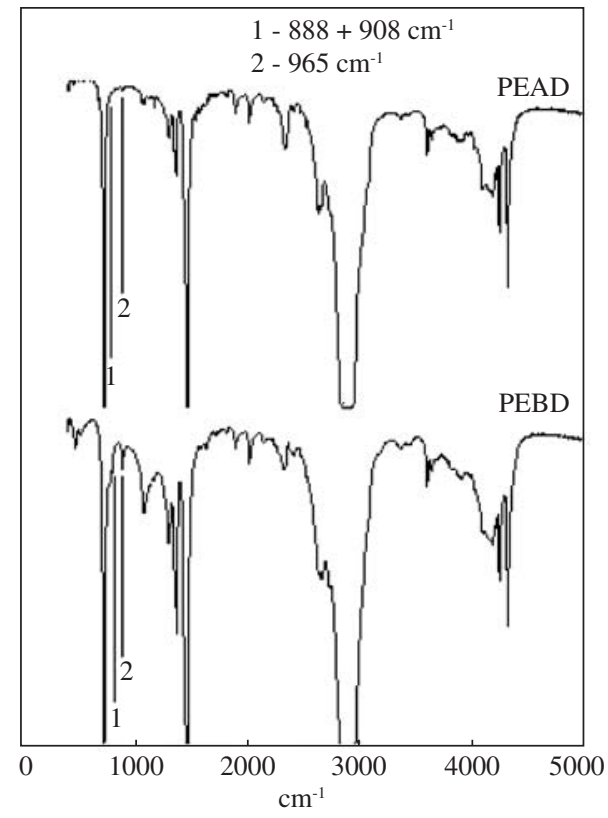

Figura 3. Espectro de FTIR do PEBD e do PEAD. lisadores comerciais de FCC. Este resultado confirma a maior susceptibilidade das ramificações existentes nas cadeias poliméricas de PEBD, ao craqueamento catalítico, produzindo maior percentagem de moléculas na faixa da Gasolina, como mostrado na Figura 5. Observa-se também que as maiores concentrações de PEBD na carga combinada (6 e 10\% p/p) produzem maiores percentuais das frações de Diesel, na utilização do catalisador comercial de baixa atividade (BA), muito provavelmente devido à baixa eficiência deste tipo de catalisador em quebrar uma maior quantidade de cadeias principais de PEBD,

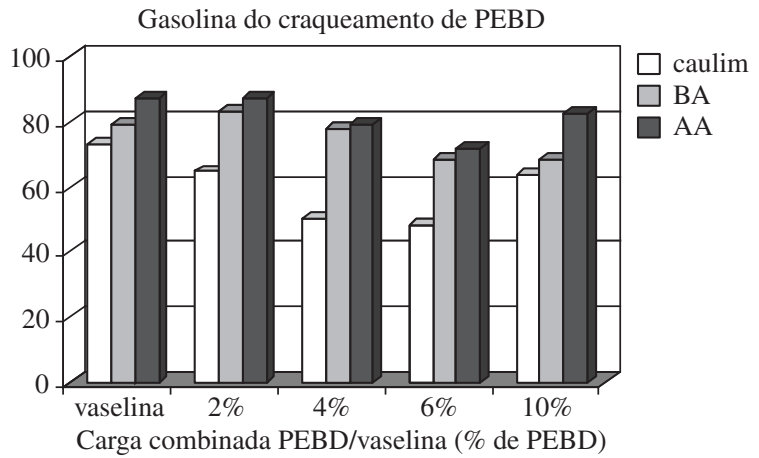

Figura 4. Percentual de produtos obtidos na faixa da Gasolina, a partir do craqueamento catalítico da vaselina e das cargas combinadas de PEBD/vaselina, frente ao caulim e aos catalisadores comerciais de FCC.

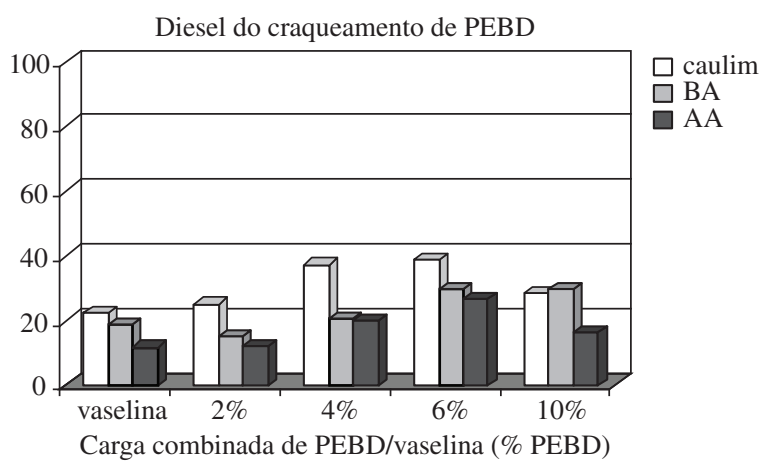

Figura 5. Percentual de produtos obtidos na faixa do Diesel, a partir do craqueamento catalítico da vaselina e das cargas combinadas de PEBD/vaselina, frente ao caulim e aos catalisadores comerciais de FCC.

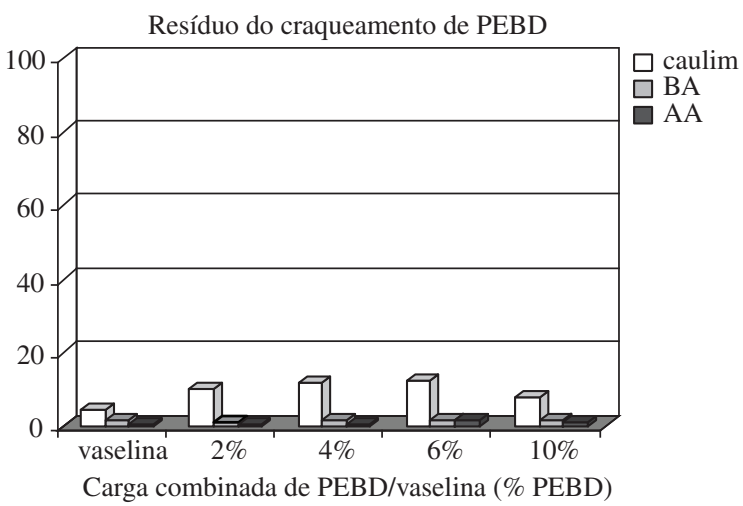

Figura 6. Percentual de produtos obtidos na faixa do Resíduo, a partir do craqueamento catalítico da vaselina e das cargas combinadas de PEBD/vaselina, frente ao caulim e aos catalisadores comerciais de FCC. 
para produção da fração de Gasolina. Em contrapartida, observa-se que o catalisador comercial de alta atividade (AA) é o que produz menores percentuais da fração Diesel, corroborando com os resultados já observados na Figura 5.

Para a produção da fração Resíduo (Figura 6), o caulim (material inerte) também se mostrou mais eficiente para todas as concentrações de PEBD, indicando que houve mais craqueamento térmico, com a utilização desse material. Os catalisadores comerciais de FCC, em contrapartida, produziram traços percentuais da fração Resíduo.

Os percentuais dos produtos líquidos do craqueamento da vaselina e das cargas combinadas de PEAD/vaselina (2 e $4 \% \mathrm{p} / \mathrm{p}$ ), na presença de caulim e dos catalisadores comerciais de FCC estão apresentados nas Figuras 7-9. A Figura 7 mostra que as cargas combinadas de PEAD/vaselina (2 e 4\% p/p) não atingiram percentuais de produção da fração Gasolina equivalentes aos percentuais atingidos pelo PEBD, nas mesmas concentrações, para nenhum dos 2 tipos de catalisadores comerciais de FCC. Este resultado mostra a dificuldade das moléculas de PEAD em serem craqueadas pelos catalisadores comerciais de FCC. Essa dificuldade é atribuída à alta cristalinidade desse polímero. No entanto, nas duas concentrações de PEAD, o catalisador comercial de baixa atividade (BA) é eficiente o suficiente para produzir alto percentual da fração Gasolina, muito provavelmente decorrente do craqueamento das poucas ramificações existentes no PEAD.

Pode-se observar pela Figura 8 que a produção da fração Diesel obtida pelo craqueamento do PEAD, atinge maiores percentuais na presença de caulim (material inerte), indicando que a produção dessa fração se dá preferencialmente através de craqueamento térmico. As cargas combinadas (2 e $4 \% \mathrm{p} / \mathrm{p}$ ) apresentam maiores percentuais dessa fração com a utilização do catalisador comercial de alta atividade (AA). Quando comparado ao PEBD, nestas mesmas concentrações, as cargas combinadas de PEAD/vaselina apresentaram um ligeiro aumento na produção da fração Diesel.

O comportamento das cargas combinadas de PEAD/vaselina para produção da fração Resíduo (Figura 9) é semelhante ao observado para as cargas combinadas de PEBD/vaselina.

\section{Análise do produto líquido por espectroscopia na região do infravermelho (FTIR)}

A análise de FTIR também foi utilizada para analisar os produtos líquidos das reações de craqueamento. Foram analisados todos os produtos líquidos obtidos pelas reações de craqueamento da vaselina pura e das misturas com PEBD e PEAD, na presença dos catalisadores de FCC.

As Figuras 10 e 11 apresentam os espectros de FTIR dos produtos líquidos do craqueamento da vaselina e das cargas combinadas dos polímeros PEBD e PEAD, nas concentrações ( 2 e $4 \%$ p/p), frente aos catalisadores comerciais de FCC.

Os espectros de FTIR comprovaram que ocorreu craqueamento catalítico e não somente craqueamento térmico, uma vez que os espectros dos produtos líquidos obtidos pelo craqueamento catalítico dos polímeros (PEBD e PEAD), na

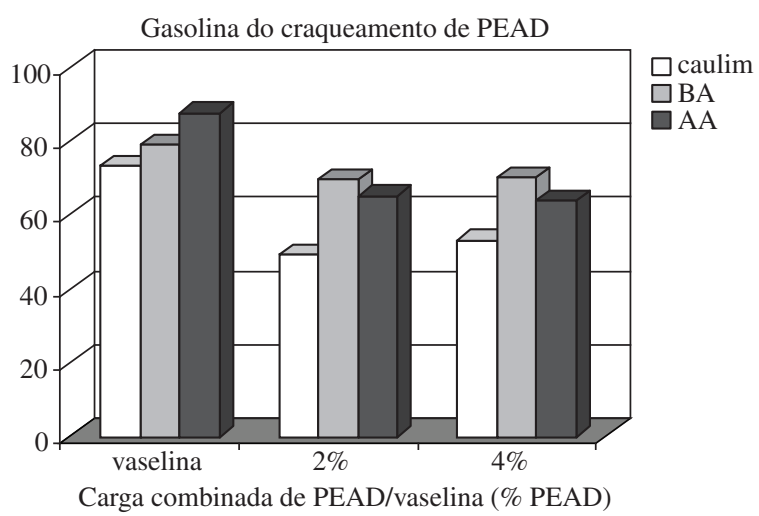

Figura 7. Percentual de produtos obtidos na faixa da Gasolina, a partir do craqueamento catalítico da vaselina e das cargas combinadas de PEAD/vaselina, frente ao caulim e aos catalisadores comerciais de FCC.

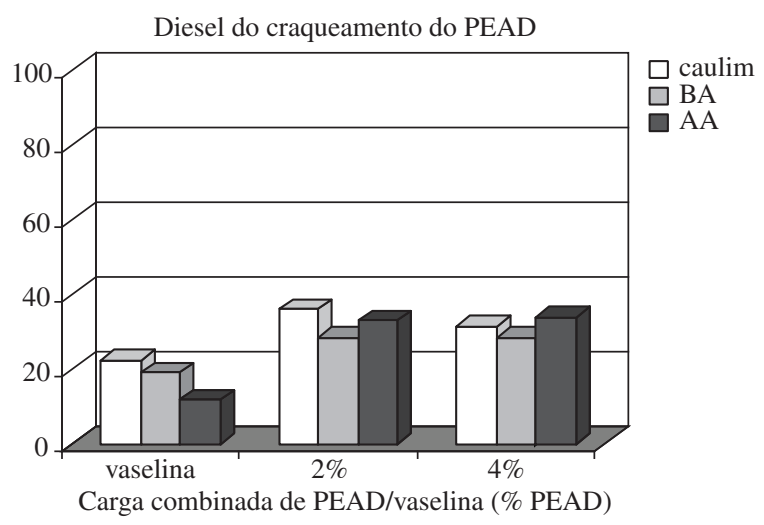

Figura 8. Percentual de produtos obtidos na faixa do Diesel, a partir do craqueamento catalítico da vaselina e das cargas combinadas de PEAD/vaselina, frente ao caulim e aos catalisadores comerciais de FCC.

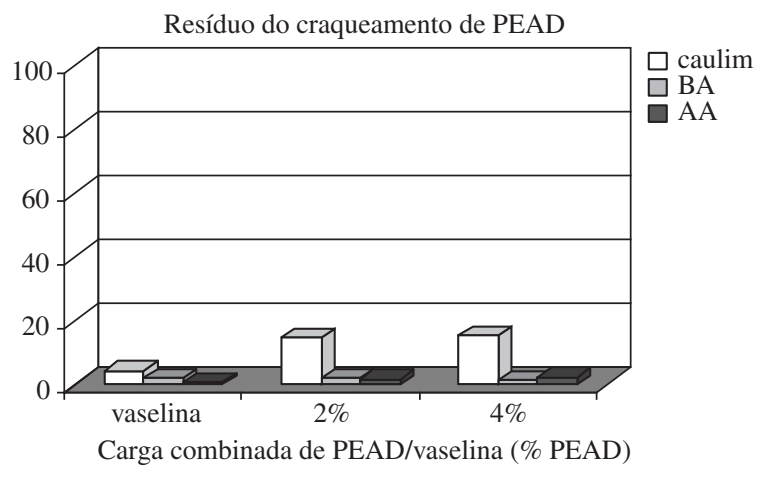

Figura 9. Percentual de produtos obtidos na faixa do Resíduo, a partir do craqueamento catalítico da vaselina e das cargas combinadas de PEAD/vaselina, frente ao caulim e aos catalisadores comerciais de FCC.

presença dos catalisadores comerciais de FCC apresentaram diferenças perceptíveis dos espectros das cargas combinadas na presença de caulim, utilizado como material inerte.

Os espectros da vaselina (mistura de parafinas) também apresentaram algumas diferenças em relação ao espectro do produto líquido obtido pelo craqueamento da vaselina pura com caulim, indicando a ocorrência de craqueamento catalítico e a eficiência dos catalisadores de FCC. Os produtos 


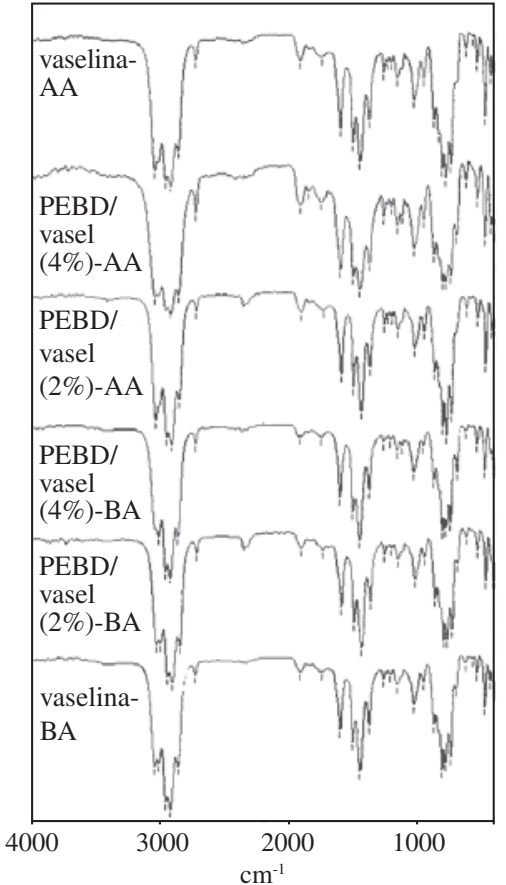

Figura 10. Espectro de FTIR do produto líquido do craqueamento da vaselina e da carga combinada PEBD/vaselina ( 2 e $4 \% \mathrm{p} / \mathrm{p})$, frente aos catalisadores comerciais de FCC (BA e AA).

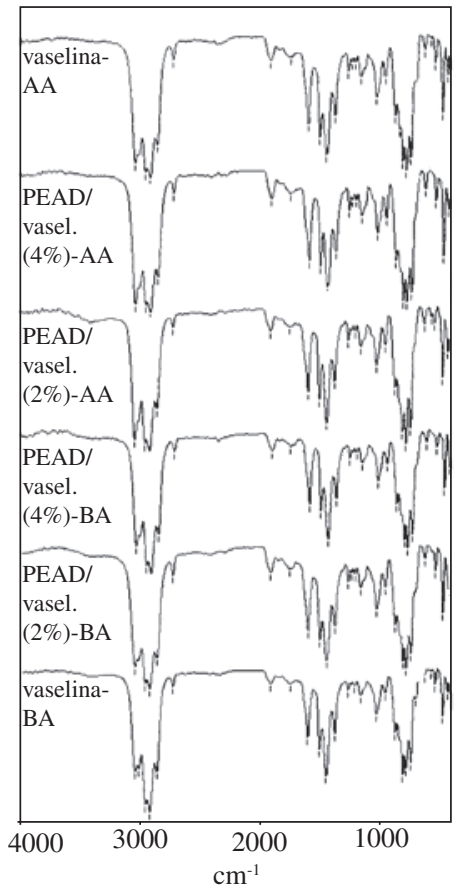

Figura 11. Espectro de FTIR do produto líquido do craqueamento da vaselina e da carga combinada PEAD/vaselina (2 e 4\% p/p), frente aos catalisadores comerciais de FCC (BA e AA).

líquidos do craqueamento das cargas combinadas dos polímeros (PEBD e PEAD), na presença de caulim e dos catalisadores também apresentaram espectros de FTIR diferentes, indicando que também ocorreu craqueamento catalítico dos polímeros.
Ao se comparar o espectro de FTIR do padrão de FCC com os espectros de FTIR dos produtos líquidos da vaselina e das cargas combinadas, na presença de caulim, foram observadas muitas semelhanças. Os produtos líquidos obtidos na presença dos catalisadores de FCC (BA e AA) apresentaram espectros semelhantes, somente com variação na intensidade de suas bandas. A presença do catalisador provavelmente provocou a aromatização de algumas cadeias, verificada pela presença de bandas existentes na região de deformação axial de C-H de aromático, como apresentado pelo espectro do xileno.

\section{Determinação de coque do produto sólido}

A análise de determinação de coque no produto sólido mostrou que, quanto maior a atividade do catalisador maior a formação de coque, depositada tanto na superfície do catalisador como no interior de seus poros, desativando-o completamente.

Os resultados obtidos para a percentagem de coque por carga indicaram que houve grande formação de coque na presença do catalisador de alta atividade, uma vez que os valores de referência da FCC S.A. devem estar entre 3\% e no máximo $6 \%$. Acima de $6 \%$ já é considerado muita formação de coque e a corrida é repetida. A Figura 12 mostra os valores de percentagem coque por carga correspondente ao percentual de produto sólido obtido após craqueamento (produto sólido produzido).

\section{Quantidades do produto gasoso por diferença com os resultados do produto líquido e do produto sólido}

As quantidades de produtos gasosos foram obtidas pela diferença do somatório dos produtos (líquido + sólido). Para a determinação do percentual de produto gasoso gerado, foi calculado primeiramente, o percentual de produto líquido, a partir da Equação 2, uma vez que o percentual de produto sólido já havia sido calculado. Os resultados dos produtos gasosos estão apresentados na Figura 13.

Os valores obtidos estão bastante coerentes. A baixa percentagem de produtos líquidos, obtidos a partir do craqueamento catalítico na presença do catalisador comercial de alta atividade contrapõe com as altas percentagens de produtos

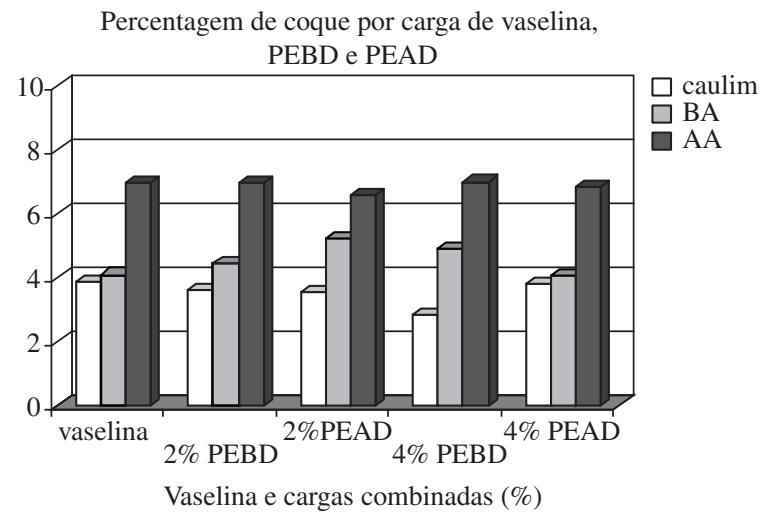

Figura 12. Percentual de produtos sólidos (coque), a partir do craqueamento catalítico da vaselina e das cargas combinadas de PEBD e PEAD, frente ao caulim e aos catalisadores comerciais de FCC. 
Percentagem dos produtos do craqueamento da vaselina, PEBD e PEAD

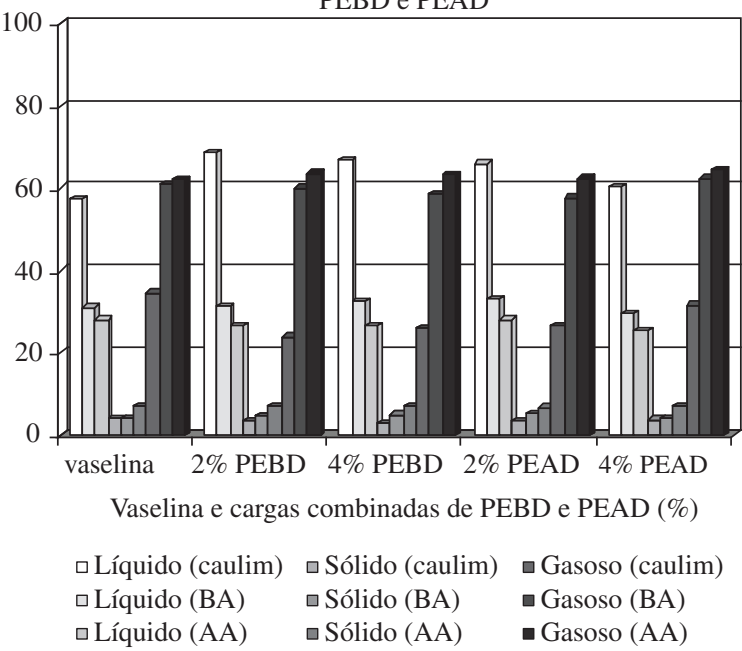

Figura 13. Percentagem dos produtos líquidos, sólidos e gasosos produzidos pelo craqueamento da vaselina e dos polímeros PEBD e PEAD.

gasosos produzidos na presença deste mesmo catalisador. Por outro lado, a alta percentagem de produtos líquidos obtidos na presença do caulim (material inerte) indica a quebra de cadeias poliméricas por craqueamento térmico, e a diminuição de produtos gasosos nas mesmas condições.

\section{Conclusões}

O craqueamento catalítico de cargas combinadas de misturas de polímeros (PEBD e PEAD) com vaselina, utilizando catalisadores comerciais de FCC de diferentes atividades (baixa e alta), proporcionou bons resultados para a produção de frações combustíveis, dependendo diretamente dos catalisadores de FCC.

$\mathrm{O}$ craqueamento catalítico das cargas combinadas de PEBD/vaselina (2, 6 e $10 \%$ p/p), na presença do catalisador comercial de FCC de alta atividade (AA), produz uma maior percentagem da fração Gasolina, mostrando a eficiência deste catalisador, provavelmente devido à ocorrência de quebra tanto de ramificações, quanto de cadeias principais deste polímero. As produções das frações Diesel e Resíduo foram favorecidas pela presença do material inerte, mostrando a prevalência de craqueamento térmico.

Para o craqueamento das cargas combinadas de PEAD/vaselina, o catalisador de menor atividade (BA) se mostrou mais eficiente para a produção da fração Gasolina, enquanto que para a obtenção das frações Diesel o catalisador de alta atividade (AA) se mostrou tão eficiente quanto o material inerte, indicando que ocorreu tanto craqueamento catalítico, quanto térmico. As frações Resíduo são produzidas preferencialmente por craqueamento térmico, na presença de material inerte.

O craqueamento das cargas combinadas de misturas de PEBD com vaselina pode ter sido facilitado devido ao PEBD, possivelmente, apresentar muitas ramificações, além de possuir um peso molecular numérico médio (Mn) menor. Sua temperatura de degradação térmica também é inferior ao do PEAD, e apresenta temperatura de solubilização menor. Todos esses pontos podem ter favorecido o craqueamento do PEBD, em comparação ao PEAD.

\section{Referências Bibliográficas}

1. Coutinho, F. M. B.; Mello, I. L. \& De Santa Maria, L. C. - Polímeros: Ciência e Tecnologia, 13, p. 1 (2003).

2. Doak, K. W. - "Ethylene Polymers", in: Encyclopedia of Polymer Science and Engineering, John-Wiley \& Sons (ed.), vol. 6, New York (1986).

3. Billmeyer, F. W. J. - "Textbook of Polymer Science", Wiley-Interscience, USA (1984).

4. Pereira, R. A.; Mano, E. B.; Dias, M. L. \& Acordi, E. B. - Polym. Bull., 38, p. 707 (1997).

5. Abadie, E. - "Craqueamento Catalítico", CENPES - DIVEN (1985).

6. Lee, S. Y.; Yoon, J. H.; Kim, J. R. \& Park, D. W. - Polym. Degrad. Stab., 74, p. 297 (2001).

7. Lee, K. H.; Noh, N.; Shin, D. H. \& Seo, Y. - Polym. Degrad. Stab., 78, p. 539 (2002).

8. Songip, A. R.; Masuda, T.; Kuwahara, H. \& Hashimoto, K. - Appl. Catal. Bull., 2, p. 153 (1993).

9. Ukei, H.; Hirose, T.; Horikawa, S.; Takai, Y.; Taka, M.; Azuma, N. \& Ueno, A. - Catal. Today, 62, p. 67 (2000).

10. Audisio, G.; Bertini, F.; Beltrame, P. L. \& Carniti, P. - Makromol. Chem., Macromol. Symp., 57, p. 191 (1992).

11. Beltrame, P. L.; Carniti, P.; Audisio, G. \& Bertini, F. - Polym. Degrad. Stab., 26, p. 209 (1989).

12. Lin, R. \& White, R. L. - J. Appl. Polym. Sci., 58, p. 1151 (1995).

13. Marcilla, A.; Gómez, A.; Reyes-Labarta, J. A.; Giner, A. \& Hernández, F. - J. Anal. Appl. Pyrol., 00, p. 1 (2003).

14. Seo, Y. H.; Lee, K. H. \& Shin, D. H. - J. Anal. Appl. Pyrol., 00, p. 1 (2002).

15. Serrano, D. P.; Aguado, J.; Escola, J. M. \& Garagorri, E. - J. Anal. Appl. Pyrol., 58-59, p. 789 (2001).

16. Vasile, C.; Onu, P.; Bãrboiu, V.; Sabliovschi, M. \& Moroi, G. - Acta Polymerica, 36, p. 543 (1985).

17. Vasile, C.; Onu, P.; Bãrboiu, V.; Sabiovschi, M.; Moroi, G.; Gânju, D. \& Florea, M. - Acta Polymerica, 39, p. 306 (1988).

18. Giannetto, G. P. - "Zeólitas - Características, Propriedades y Aplicaciones Industriales", Ediciones Inovación Tecnológicas (ed), Caracas (1990).

19. Pujadó, P. R.; Rabó, J. A.; Antos, G. J. \& Gembicki, S. A. - Catal. Today, 13, p. 113 (1992).

20. AMERICAN SOCIETY FOR TESTING AND MATERIALS, MÉTODO ASTM D 3907-92, Standard Test Method for Testing Fluid Catalytic Cracking (FCC) Catalysts by Microactivity Test.

21. Ribeiro, A. M. - "Craqueamento catalítico de cargas combinadas de polímeros utilizando catalisadores comerciais de FCC", Dissertação de Mestrado, Departamento de Engenharia Química, Instituto de Tecnologia, Universidade Federal Rural do Rio de Janeiro, Rio de Janeiro, Brasil (2004)

22. Rocha, F. C. - "Síntese de poliolefinas com ramificações de cadeia longas através de catalisadores metalocênicos", Dissertação de Mestrado, Instituto de Macromoléculas Professora Eloisa Mano, Universidade Federal do Rio de Janeiro, Rio de Janeiro, Brasil (2004).

23. Vandermiers, C.; Moulin, J. F.; Damman, P. \& Dosière, M. Characterization of molecular heterogeneities of LLDPE by multiple crystallization-dissolution steps. Polymer, 41, p. 2915-2923 (2000).

Enviado: 15/05/06

Reenviado: 09/08/06

Aprovado: 20/08/06 